\title{
PERCEPCIÓN DEL PERSONAL DE ENFERMERÍA FRENTE A LOS PROGRAMAS \\ DE SEGURIDAD DEL PACIENTE
}

\section{Autor: Deysi Lizeth Cárdenas Pinzón}

\section{RESUMEN:}

El presente artículo tiene como objetivo principal realizar un proceso critico investigativo sobre de la literatura que existe en las diferentes bases de datos lilacs, pubmed, scielo, scopus, science direct, ebsco, con respecto a la precepción que tienen el personal de Enfermería frente a los diferentes programas de seguridad del paciente, por tal motivo se tomaron como referencia 50 publicaciones que cumplían con los criterios de inclusión. La muestra final de esta revisión fueron 45 artículos citados en el estudio, que permitieron la estructura y análisis de dos categorías: percepción punitiva: factor que genera en el personal, en el miedo al reporte y percepción de sobrecarga laboral: que influye como barrera para el mejoramiento del cuidado de las personas, generando como conclusión principal la importancia de fortalecer la cultura del reporte, e identificar oportunidades de mejora que permitan un cuidado de calidad hacia los pacientes, y por ende se evidencie una mejora en la calidad de la prestación de los servicios de salud.

Palabras clave: Enfermería, percepción, seguridad del paciente.

\begin{abstract}
:
The main objective of this article is to conduct a critical investigative process on the literature that exists in the different databases lilacs, pubmed, scielo, scopus, science direct, ebsco, with respect to the perception that Nursing staff have about the different patient safety programs, for this reason, 50 publications that met the inclusion criteria were taken as reference. The
\end{abstract}


final sample of this review were 45 articles cited in the study, which allowed the structure and analysis of two categories: punitive perception: factor that generates in the personnel, in the fear of reporting and perception of work overload: that influences as a barrier to the improvement of the care of the people, generating as main conclusion the importance of strengthening the culture of the report, and identifying improvement opportunities that allow a quality care towards the patients, and therefore evidencing an improvement in the quality of the provision of health services.Keywords: Nursing, perception, patient safety.

\section{Introducción:}

El personal de Enfermería es el responsable de una gran cantidad de efectos sobre los pacientes ya sean de carácter positivo o negativo, dado que son el responsable directo del cuidado integral que se brinda a la persona con respecto a su salud. Un estudio realizado 2004 por el Institute of Medicine (IOM) de los Estados Unidos refieren que el personal de enfermería trabaja en un ambiente caracterizado por múltiples factores que predisponen a eventos asociados al cuidado ${ }^{6}$, tales como: ulceras por presión, administración de medicamentos, enfermedades nosocomiales entre otras ${ }^{278}$. Esto debido al incremento en la demanda en los servicios de salud y al poco personal que se dispone para tal fin, además de factores ambientales estructurales y de índole administrativo que también dificultan el que hacer enfermero, muchas veces están obligados a suplir las necesidades de los pacientes con muy escasos recursos imposibilitando un cuidado de calidad, pues existen muy pocos recursos en los establecimientos de salud y de personal asistencial, esto aunado a las grandes exigencias que se imparten a nivel administrativo para el cumplimiento de metas respecto a la cobertura de salud, haciendo a un lado la calidad en la prestación de los servicios.

En el hacer del enfermero o enfermera ya sea profesional o auxiliar, se encuentra inmerso en el concepto de seguridad del paciente y hace parte de su esencia, la OMS define a esta 
profesión como: “una profesión con profundas raíces humanistas, genuinamente preocupada por las personas que confían en su cuidado"9 , es así que los enfermeros y enfermeras son de vital importancia para el cumplimiento y el empoderamiento de los programas de seguridad del paciente pues no solo están inmersos en la administración de los medicamentos o en los cuidados básicos de la persona sino que también se preocupa por su bienestar mental integrando su salud física y mental.

Por tal motivo se identifica la necesidad de realizar un proceso crítico investigativo de la literatura sobre la percepción que tiene el personal de enfermería frente a los programas de seguridad del paciente, para así evidenciar una realidad frente a las necesidades que expresan los trabajadores, respecto a los programas. Empoderando una cultura de seguridad del paciente que permita al personal de enfermería identificar necesidades en su entorno laboral, y situaciones de riesgo que se pueden prevenir, generando confianza en el personal para reportar y analizar los potenciales casos de riesgos, evidenciando a su vez las oportunidades de mejora y convirtiendo estas oportunidades en un sistema de salud más amigable e integral con el usuario.

Los programas de seguridad del paciente implementan actividades y diseñan herramienta que permiten el mejoramiento de la calidad de los servicios, (reuniones briefing, listas de verificación, protocolos de prácticas seguras, incidencias de efectos adversos, etc.) ${ }^{10}$ pero estas actividades son poco conocidas debido a la escasa documentación que se genera a partir de las experiencias exitosas que se llevan a cabo con las mismas, disminuyendo la posibilidades de mejora en otros ámbitos de las salud y el impacto que estas actividades y herramientas tienen sobre el personal de enfermería, pero sobretodo en la persona objeto de cuidado. 
Por lo anterior se hace necesario realizar por medio de presente artículo un revisión sistemática e integrativa de literatura científica ya existente, que permita reconocer y analizar las necesidades del personal de enfermería frente a los programas de seguridad del paciente, como base para la construcción de herramientas que mejoren la planeación del cuidado, que fomenten la apropiación de los programas de seguridad del paciente como una cultura inmersa en el ejercicio ético-laboral del personal de enfermería y así disminuir la probabilidad de eventos asociados al cuidado.

\section{MATERIALES Y MÉTODOS}

Revisión de bases de datos LILACS, PUBMED, SciELO, SCOPUS, SCIENCE DIRECT, EBSCO; Para guiar la revisión de artículos se formuló la siguiente pregunta ¿Cuál es la percepción del personal de enfermería frente a los programas de seguridad del paciente?; Los criterios para la inclusión de artículos para esta revisión fueron artículos publicados en inglés, portugués y español, con resúmenes disponibles en bases de datos seleccionados en el período comprendido 2000 al 2018; Como criterios de exclusión fueron los estudios que no tenían ninguna relación con los conceptos, métodos y propósito de este estudio.

Se inició la revisión con la identificación de las fuentes documentales, recopilación de información y posterior análisis, para el reconocimiento de las ideas sobre el objeto de estudio. La búsqueda se realizó tomando como base los Descriptores en Ciencias de la Salud (DeCS): percepción de enfermería sobre seguridad del paciente, cultura de seguridad del paciente, calidad en salud. Tras la identificación de los contenidos incluidos en las bases de datos, se limita la búsqueda a los artículos que traten el tema.

El análisis de datos se realizó en dos etapas: en el primer paso se identificaron datos de ubicación del artículo, autor, objetivos, metodología, resultados principales, el cumplimiento del estudio basado en la pregunta de revisión y objetivo. En el segundo paso, se analizan los 
artículos cuyos resultados se resumen en el contenido del concepto, método, finalidad y resultados.

Es así que se analizaron y discutieron publicaciones realizadas, con el fin de hacer un análisis reflexivo sobre su contribución a la construcción del conocimiento disciplinar, que genere herramientas para la planeación del cuidado y fomentar el mejoramiento de la calidad de los servicios de salud por parte del personal de Enfermería.

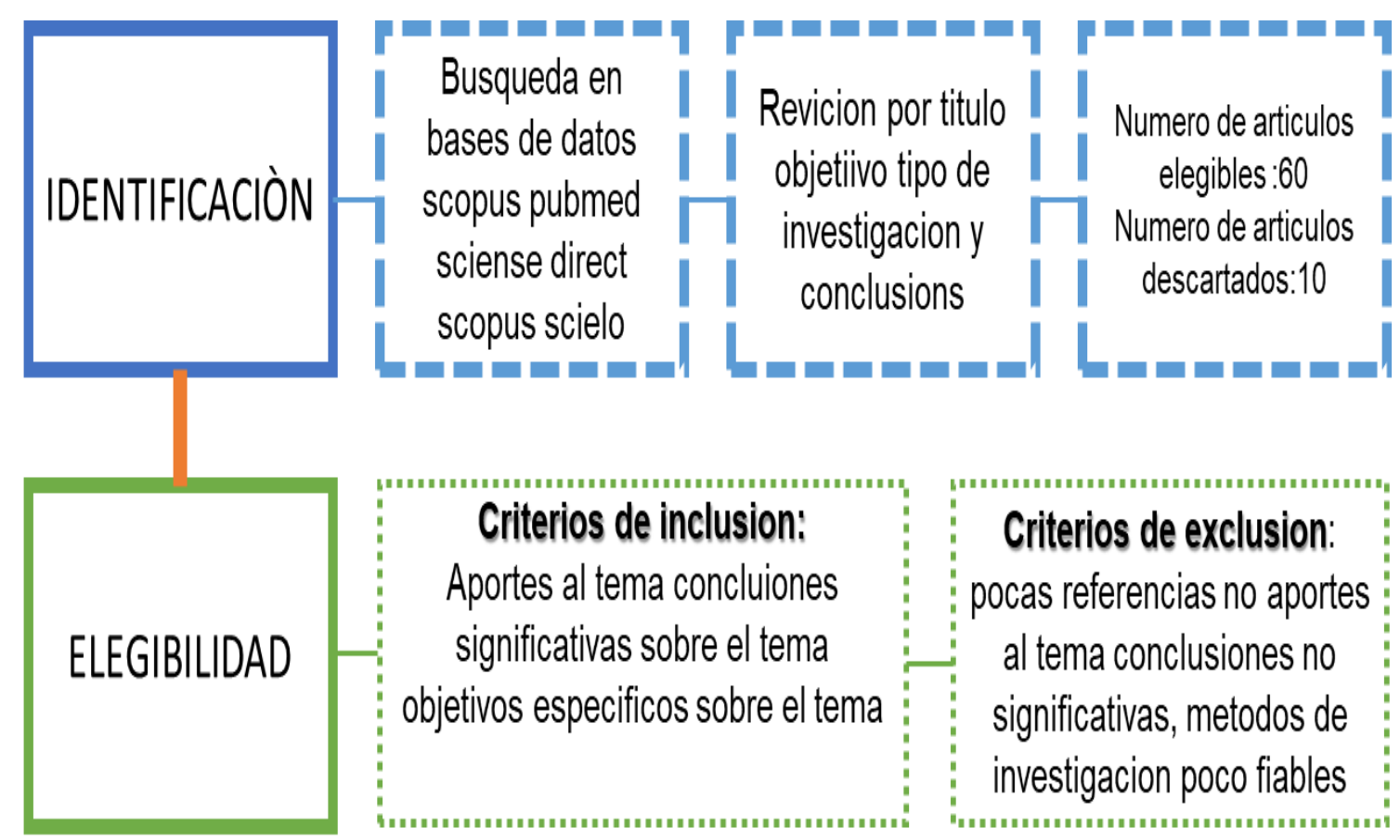

\section{RESULTADOS:}

Se identificaron 50 estudios potenciales y se realizó la lectura exploratoria para la selección de textos para el análisis; La muestra final de esta revisión fueron 45 artículos citados en el estudio, que permitieron la estructura y análisis de dos categorías: percepción punitiva: factor que genera en el personal, en el miedo al reporte y percepción de sobrecarga laboral: que influye como barrera para el mejoramiento del cuidado de las personas.

\section{Percepción punitiva.}


El personal de Enfermería, enfoca su quehacer en el cuidado integral de la persona teniendo en cuenta todas sus dimensiones, y procura porque cada una de estas dimensiones este siendo tratada para el completo bienestar del sujeto de cuidado, es así que en la mayoría de ocasiones el personal de enfermería percibe su cuidado hacia el usuario, como bueno o excelente, haciendo a un lado su esencia como ser humano, el cual se encuentra ligado al error en cualquier actividad ${ }^{11}$, en la mayoría de veces, el miedo al error disminuye la iniciativa al reporte de eventos asociados al cuidado, propios del personal de enfermería, que más allá de ser profesionales son personas que están predispuestas por miles de causas al error. Esto hace que se perciban a los programas de seguridad del paciente como unos entes juzgadores que podrían poner en peligro su estabilidad laboral.

Esta situación ha influido en que hoy en día, este sea uno de los temas más álgidos y de mayor importancia no solo en las instituciones prestadoras de salud sino en todos los entes encargados de la administración de los servicios de salud ${ }^{12}$, muchas son las personas que se han visto afectadas con la ocurrencia de eventos adversos en salud, tan solo en los EUA a mediados de los 90 se informó que: “entre 44000 a 98000 personas mueren al año en los hospitales de ese país, como resultado de errores que se suceden en el proceso de atención"13 y nos solo estamos hablando de los pacientes como sujetos de cuidado, sino del mismo personal de salud el cual también se ve $\operatorname{afectado}^{14}$ y encasillado como el culpable de que estos eventos sucedan, ya que se encuentran en constante contacto con las personas haciéndolos responsables directos del cuidado de las mismas ${ }^{15}$, sin tener en cuenta que existen muchos factores que inciden en que se presente o no un evento asociado al cuidado.

Muchas veces algunos programas se muestran con una función total mente diferente a la de su esencia, pues son percibidos con un objetivo punitivo frente al evento y no busca la causa fundamental o de base de la ocurrencia de eventos ${ }^{31617}$. sino que, por el contrario, buscan específicamente un culpable que justifique todo el suceso, esto genera en el personal 
un miedo inminente a ser juzgado, y evita que se propicien oportunidades de mejoramiento, que en algunas ocasiones pueden evidenciar falencias del mismo sistema operativo de la institución prestadora de servicios de salud. "Ser recompensado por relatar un problema de seguridad del paciente aumenta las posibilidades de que los profesionales hagan esto otras veces, mientras que si fue castigado, disminuye esa posibilidad"18, esta recompensa puede significar para las entidades prestadoras de salud una solución efectiva e integral a muchas de las barreras que se presentan en la ejecución del cuidado, además genera una confianza en el personal que le permite identificarse como ser humano predispuesto a cometer errores y también capaz de mejorar y ser abanderado de los procesos de calidad de las instituciones. ${ }^{19}$

Por lo dicho anteriormente, la mayoría de la literatura encontrada sugiere que es necesario, un mayor compromiso administrativo pues no solo se debe buscar el error humano sino ver el trasfondo y la realidad que sucede al rededor del evento. ${ }^{2021}$ Giraldo y Montoya encontraron en su estudio evaluacion de la cultura de seguridad del paciente en un hospital de Antioquia Colombia, que la falta de interés por llegar al trasfondo de los eventos por parte de los programas de seguridad del paciente es una de las percepciones más sentidas por el personal de enfermería ya que propicia la sensación sancionatoria y no de apoyo para incentivar la calidad de los servicios.

"Fomentar la cultura del reporte y aprender de los errores deben ser prioridades en los hospitales de todo el mundo" 22 . Sánchez Izquierdo y colaboradores afirman en su investigación que los programas de seguridad del paciente, que se comprometieron con la constante capacitaron al personal sobre cultura de seguridad del paciente y su importancia en los procesos de salud enfermedad, tenían una mayor aceptación he hicieron que la percepción de los mismos fuera más positiva ${ }^{10}$, en otros estudios también se evidenciaron mejoras en la calidad de la prestación de los servicios de salud, generando en los usuarios una percepción de seguridad frente al personal de enfermería, al cual se encontraba a cargo su cuidado ${ }^{223}$. 
Por lo tanto, se hace necesario que todo el personal se encuentre en continua capacitación sobre el tema, para que así mismo se entiendan los programas de seguridad del paciente como un apoyo gestor de la calidad del cuidado, el cual se alimenta día a día con ideas, compromisos y tareas que surgen desde el mismo personal y así incentivar el inicio de la seguridad del paciente como cultura. ${ }^{24}{ }^{25}$

Realizar un análisis minucioso de cada uno de los factores que influyen en un evento asociado al cuidado, crea estrategias, y genera oportunidades de mejoramiento, tanto en la calidad del personal asistencial en el concepto de seres integrales expuestos a cometer errores, como en la calidad de los servicios de salud, todo esto influye en una mejor atención y en la disminución de costos que se generen por una atención deficiente, creando ambientes seguros para todos los usuarios, generando confianza en los trabajadores, y empoderándolos en todos y cada uno de los procesos de calidad que promueven una cultura de seguridad del paciente.

\section{Percepción de sobrecarga laboral.}

Con el pasar de los tiempos y la implementación de nuevas tecnologías que permiten ser más precisos en el diagnóstico de las patologías y el tratamiento de las mismas, haciendo que estos tengan un carácter diferencial específico para cada persona, ha crecido también la responsabilidad no solo del personal médico quienes tienen el compromiso de un diagnóstico y tratamiento adecuado oportuno y eficaz, sino mucho más del personal de enfermería quienes son los que están en una mayor proporción de tiempo encargados del cuidado directo del paciente, haciendo por ende que exista una mayor demanda de servicios, pero no así la cantidad de personal para suplirlos, esta demanda requiere de un equipo multidisciplinar que procure un cuidado integral, holístico de la persona que no depende tan solo del personal de Enfermería, a pesar de que este parezca ser el único implicado. ${ }^{26}$ 
Uno de los puntos de vista más comunes dentro del personal de Enfermería durante la revisión bibliográfica fue la sobrecarga laboral la cual, según la percepción de los mismos incrementa la posibilidad de incidir en eventos que afectan tanto la salud del paciente, como la salud del trabajador ${ }^{27}$ las largas jornadas laborales ${ }^{28} \mathrm{y}$ la gran cantidad de pacientes a su cargo, generan una sensación de sobrecarga en el personal de Enfermería ya sea profesional o tecnico ${ }^{20}$.

Muchas veces las cargas laborales a las que se enfrenta el personal de Enfermería, superan en gran cantidad la capacidad física del mismo, produciendo en ellos un desgaste físico y mental que aumenta la posibilidad del error en el cuidado, todo esto sumado a las exigencias por parte de un sistema económico que mide cuantitativamente la efectividad de los servicios, pero no brinda apoyo económico y estructural para a efectividad del cumplimiento de los servicios de salud, haciendo que la persona objeto de cuidado se sienta cada vez más desconfiada de asistir a las entidades de salud, generando una visión completamente errónea de todos los profesionales de la salud. ${ }^{29}$

En gran cantidad de la literatura encontrada se sugiere que los programas de seguridad del paciente deben ser multidisciplinares, pero cabe destacar que el liderazgo se encuentra a cargo de los profesionales de enfermería ${ }^{30}$. Esto conlleva a que todo el personal de salud debe estar debidamente coordinado y dispuesto a seguir los lineamiento ya establecidos por el Ministerio de Protección Social ${ }^{31}$, no como un fenómeno impuesto sino más bien como una cultura que tiene en su trasfondo en el deber ser de todo el personal de salud, el cual es el mejoramiento de la salud de las personas ${ }^{1}$.

En un estudio realizado por Escobar y colaboradores muestra que muchas veces la percepción del dolor del paciente es más significativa, cuando el entorno laboral del 
enfermero no es favorable en diferentes sentidos, como por ejemplo con la falta de recursos tanto físicos como estructurales y humanos, reflejando una percepción negativa de la calidad de cuidado que tienen los pacientes frente al personal de Enfermería; también establece "que a menor ratio enfermera/paciente, los resultados en los pacientes son mejores". ${ }^{32}$

En instituciones prestadoras de salud como las unidades de cuidado critico donde existía un número suficiente de personal de enfermería por paciente se evidencio una menor proporción de eventos adversos ${ }^{33}{ }_{-}^{34}{ }_{-}$.

Resulta claro que en la medida en que exista deficiencia en la cantidad y calidad del personal de enfermería, acompañado de sobrecarga laboral y escasos recursos de insumos médicos e insumos estructurales, estos factores se convierten en baches o barreras, que no permiten la adecuada gestión del cuidado por parte del personal de Enfermería. ${ }^{24}$

Un adecuado clima laboral mejora el desempeño del personal de Enfermería y disminuye la probabilidad de presentar eventos adversos ${ }^{35}$, el cuidado integral de la persona, está a cargo de todo el equipo multidisciplinar, que influye en el mejoramiento de la salud de las personas, desde el personal de limpieza y vigilancia, pasando por todo el equipo administrativo, hasta llegar a el equipo de salud. Todos estos son factores que influyen en el tiempo en que se produzca una mejoría en la salud del paciente, así como la disminución de costos para las entidades de salud, un equipo multidisciplinar enmarcado y empoderado con la cultura de seguridad del paciente disminuye la posibilidad de eventos asociados al cuidado, mejora la percepción del paciente frente a las entidades de salud y produce recursos que mejoran la calidad de los servicios.

\section{DISCUSIÓN}

Con el creciente desarrollo de políticas que propenden por el mejoramiento de los servicios de salud y la calidad de la atención en los servicios, muchas entidades de salud han 
adoptado programas y guías de seguridad del paciente apoyados en las guías nacionales, para que de esta forma se puedan prever y evitar muchos de los incidentes que suceden por errores humanos del mismo personal de salud. Incidentes que en su gran mayoría no son intencionales, sino que son producto de muchos factores que rodean e intervienen en el cuidado de las personas.

El personal de enfermería son quienes más se encuentran inmersos en esta situación, ya que en una medida de tiempo disponen de más contacto con el paciente y quienes debe prestar más atención a sus necesidades ${ }^{3637}$, pero también son quienes tienen más riesgo de presentar algún evento adverso, por tal motivo es de gran importancia conocer la percepción del personal de Enfermería frente a los programas de seguridad del paciente ${ }^{38}$, esto con el fin de dar herramientas que permitan a los mismos potencializar todos los esfuerzos por mejorar este tipo de programas ya que muchas de estas estrategias pueden provenir de los mismos trabajadores $^{2139}$.

Es evidente que como seres humanos muchas veces la percepción del cambio no es muy favorable sin embargo el personal de salud en su mayoría, se muestra siempre presto al cambio siempre y cuando esté a favor del cuidado del paciente, y encuentre en el programa líderes que escuchen sus dudas, inquietudes y aportes, que busque soluciones a las diferentes problemáticas que se presenten y no solamente busquen un culpable o una sanción ${ }^{26}$.

Sin embargo, la percepción del personal de enfermería frente a la sobrecarga laboral ${ }^{40}$ es muy marcada sobretodo en estudios de habla hispana ya que la mayoría expresan que muchos de los eventos que se presentan es debido a las sobrecargas labores que tienen a su cargo y que de esa manera aumenta la probabilidad de un error que afecte la salud de la persona sujeto de cuidado y así mismo las del mismo personal de enfermería ${ }^{4130424344}$. 
Es necesario realizar más estudios que midan la percepción del personal de enfermería sobre los programas de seguridad del paciente, así como realizar la documentación de todas aquellas estrategias que se implementan por medio de estos programas ya que se sabe de muchas estrategias que se implementan, pero muy pocas son documentadas o sujetas a la investigación $^{1227}$, existe muy poca literatura actual o de los últimos años que evalúen este tema en los profesionales de enfermería, y es necesaria la evaluación continua de este tipo de programas en los hospitales ya que están en constante cambio y mejoramiento y así poder compartir experiencias exitosas que apoyen el mejoramiento de la calidad de los servicios.

\section{CONCLUSIONES:}

Muchos de los programas de seguridad del paciente presentan una percepción punitiva frente al reporte, que fomenta en los profesionales no solo de enfermería sino de todo el grupo interdisciplinar, el miedo al erro y por ende se maneja un silencio profesional que en la mayoría de los acosos afecta directamente al sujeto de cuidado, es necesario que se fomente la cultura del reporte así como la adecuada socialización y búsqueda de oportunidades de mejora en los mismo, brindando estrategias que permitan el empoderamiento de los programas en todo el personal, y visualizando así también el aporte a la investigación y la ciencia de los profesionales de enfermería, quienes son los abanderados de estos tipos de programas.

Además, por medio de estas investigaciones se pueden evidenciar problemáticas de gran peso como lo es la sobrecarga laboral, y demostrar que un profesional de la salud con demasiadas tareas está más predispuesto al error y de esta manera se genera un impacto económico negativo en las entidades prestadoras de salud, generar conciencia de que un trabajador en un ambiente laboral favorable es rentable para las instituciones, puede generar cambios a favor en el cuidado directo de la salud de las personas. 
La idea de equipos interdisciplinares en el cuidado de la salud de las personas debe ser el pilar de todo sistema de salud eficiente, si una sola persona del equipo falla podría tener consecuencias fatales, para los pacientes, pero esto es vital si cada uno de los trabajadores de una entidad prestadora de salud se concientiza que su trabajo es de gran importancia y ayuda en el mejoramiento efectivo de la salud de las personas.

\section{BIBLIOGRAFÍA}

1. Ramos F, Coca SM, Abeldaño RA. Percepción de la cultura de seguridad de pacientes en profesionales de una institución argentina. Enfermería Univ. 2017;14(1):47-53. doi:10.1016/j.reu.2016.12.004

2. Rebeca C, Reyes M, Milena S, Correa A, Marcela D, Palacio G. Seguridad del paciente y su percepción Patient safety and its perception in health professionals. 2017;17(33):277-291.

3. Strategia V, Anexa S-, Rom SG, et al. medicion de la cutura de seguiridad del paciente en un hospital publico de primer nivel en el municipio de villeta. Repos Univ del rosario. 2016:45-46.

4. Palacios-barahona AU. Factores asociados a eventos adversos en pacientes hospitalizados en una entidad de salud en Colombia. CES Med. 2012;Volumen 26(1):19-28. http://www.scielo.org.co/pdf/cesm/v26n1/v26n1a03.pdf.

5. Wilson D, Redman RW, Talsma A, Aebersold M. Differences in Perceptions of Patient Safety Culture between Charge and Noncharge Nurses: Implications for Effectiveness Outcomes Research. Nurs Res Pract. 2012;2012:1-7. doi:10.1155/2012/847626 
6. Luiz RB, Simões AL de A, Barichello E, Barbosa MH. Factors associated with the patient safety climate at a teaching hospital. Rev Lat Am Enfermagem. 2015;23(5):880-887. doi:10.1590/0104-1169.0059.2627

7. INFORMES EEI 2009 MDSYPS. redultado estudio IBEAS. Minist salud Colomb. 2017;91:399-404

8. S. T, I. G. La seguridad del paciente en urgencias y emergencias / Patient safety in emergency care. An del Sist Sanit Navarra VO - 33. 2010;33:131. doi:10.4321/s113766272010000200015

9. OMS. Organización Mundial De La Salud Magnitud De Los Eventos Adversos. Vol 1.; 2001. http://www.binasss.sa.cr/seguridad/articulos/calidaddeatencion.pdf.

10. Luis F, Izquierdo S, Moreno NO, Peláez NU, Ángeles A, González R. Análisis de la cultura de seguridad del paciente en unidades extra- hospitalarias de hemodiálisis . Evolución tras la implantación de medidas. 2018;19(1):25-33.

11. Universitaria M, Unidos E, Breta G. medicina universitaria. elseivierier. 2011;13(51):69-71.

12. Gómez Ramírez OJ, Soto Gámez A, Arenas Gutiérrez A, Garzón Salamanca J, González Vega A, Mateus Galeano E. Una mirada actual de la cultura de seguridad del paciente TT - A current description of the patient safety culture TT - Uma olhada atual da cultura da segurança do paciente. Av en Enfermería. 2011;29(2):363-374. doi:10.1016/S0749-0704(02)00075-1

13. Kohn LT, Corrigan JM, Molla S. To Err Is Human.; 2000.

14. Orkaizagirre A. La cultura de seguridad del paciente de las enfermeras hospitalarias. 2016:25 
15. H PM. La vinculación ético-jurídica entre la gestión del cuidado y la gestión de riesgos en el contexto de la seguridad del paciente. 15:141-154.

doi:10.5294/aqui.2015.15.1.13

16. Jacho C. Causas del no reporte de eventos adversos en una Institución Prestadora de Servicios de Salud en Pasto - Nariño, Colombia. SECCIÓN ARTÍCULOS Orig Rev Univ Y SALUD. 2013;Vol. 15(2):187-195.

17. Ramírez-martínez ME, Pedraza-avilés AG. Cultura de seguridad y eventos adversos en una clínica de primer nivel. Enfermería Univ. 2017;14(2):111-117.

doi:10.1016/j.reu.2017.02.006

18. Rose C, Barcelos G, Danilo W, et al. Cultura de segurança : enfermagem intensivistas a percepção dos profissionais de Safety culture : The perception of intensivist nurses. Enferm Glob. 2016;(2003):220-232. doi:10.6018/eglobal.15.1.207821

19. Ramos RR, Calidgid CC. Patient safety culture among nurses at a tertiary government hospital in the Philippines. Appl Nurs Res. 2018;44:67-75. doi:10.1016/j.apnr.2018.09.007

20. Rigobello MCG, Carvalho REFL de, Guerreiro JM, Motta APG, Atila E, Gimenes FRE. The perception of the patient safety climate by professionals of the emergency department. Int Emerg Nurs. 2017;33:1-6. doi:10.1016/j.ienj.2017.03.003

21. Junior P, Claudiano O. Evaluación de la cultura de seguridad del paciente en una organización hospitalaria de un hospital universitario Avaliação da cultura de segurança do paciente na organização hospitalar de um hospital universitário Evaluation of the patient safety culture . :309-325.

22. Okuyama, J.H.H; Galvao, T.F.; Silva MT. Healthcare Professional's Perception of Patient Safetu Measured by the Hospital Survey on Patient Safety Culture: A Systematic 
Rewiew and Meta-Analysis. Hindawi Sci Worl J. 2018;2018:11.

doi:https://doi.org/10.1155/2018/9156301

23. Friese CR, Lake ET, Aiken LH, Silber JH, Sochalski J. Hospital Nurse Practice Environments and Outcomes for Surgical Oncology Patients. 2007:1145-1163. doi:10.1111/j.1475-6773.2007.00825.x

24. Ferreira Umpiérrez A, Fort Fort Z, Tomás VC. Eventos Adversos En Salud Y Cuidados De Enfermería: La Seguridad Del Paciente Desde La Experiencia Del Profesional Eventos Adversos Na Saúde E Os Cuidados De Enfermagem: a Segurança Dos Pacientes Desde a Experiência Do Profissional Adverse Events in Healt. scielo. 2015;24(2):310-315. doi:10.1590/0104-07072015000122014

25. Minuzzi AP, Salum NC, Locks MOH. Assessment of patient safety culture in intensive care from the health team's perspective . Texto e Context Enferm. 2016;25(2):1-9. doi:10.1590/0104-07072016001610015

26. Dalila AR. DE ENFERMERIA . FACTORES QUE LA AFECTAN Human resources satisfaction with laboral conditions in infirmary . Factors that affect it. Inst Super Ciencias Médicas La Habana Fac. 2005.

27. Ross C, Rogers C, King C. Safety culture and an invisible nursing workload. Collegian. 2018. doi:10.1016/j.colegn.2018.02.002

28. Linares S. Análisis de la carga laboral del personal de enfermería , según gravedad del paciente Análisis de la carga laboral del personal de enfermería, según gravedad del paciente Analysis of the workload of nursing staff, according to the. 2018;(October). 
29. Adrián M. Esnarriaga Vicente. PROPUESTA PARA LA MEDICIÓN DE CARGAS DE TRABAJO DE ENFERMERÍA EN UCI HOSPITAL UNIVERSITARIO DE DONOSTIA. upna. 2014;trabajo ma:58.

30. Freixas Sala N, Monistrol Ruano O, Espuñes Vendrell J, et al. Patient safety and nursing implication: Survey in Catalan hospitals. Enfermería Clínica (English Ed. 2017;27(2):94-100. doi:10.1016/j.enfcle.2016.03.001

31. lineamientos para la implementacion de la politica de sguridad del paciente. Minist Prot Soc. 2008;1:43.

32. Escobar-Aguilar G, Gómez-García T, Ignacio-García E, et al. Entorno laboral y seguridad del paciente: Comparación de datos entre los estudios Seneca y RN4CAST. Enferm Clin. 2013;23(3):103-113. doi:10.1016/j.enfcli.2013.03.005

33. Rocco dr. cristian, Garrido dr alejandro. Seguridad Del Paciente Y cultura de seguridad. Rev Med Clin condes. 2017;28(5):785-795. doi:785-795

34. Montenegro JH, Romero AF, Tejada PA, Olaya SX, Rubiano AM. Perceived versus Observed Patient Safety Measures in a Critical Care Unit from a Teaching Hospital in Southern Colombia. Crit Care Res Pract. 2016;2016. doi:10.1155/2016/2175436

35. Merino-Plaza MJ, Carrera-Hueso FJ, Roca-Castelló MR, Morro-Martín MD, Martínez-Asensi A, Fikri-Benbrahim N. Relationship between job satisfaction and patient safety culture. Gac Sanit. 2018;32(4):352-361. doi:10.1016/j.gaceta.2017.02.009

36. Troncoso MP, Suazo SV. Cuidado humanizado : un desafio para las enfermeras en los servicios hospitalarios. 2007;20(4):499-503. 
37. J. Silva-Fhona, b,*, S. Ramón-Cordovab, S. Vergaray-Villanuevab VP-F y RP-R. Percepción del paciente hospitalizado respecto a la atención de enfermería en un hospital público. Enfermería Univ. 2015;12(2):80-87. doi:10.1016/j.reu.2015.04.001

38. Mir-abellán R, Falcó-pegueroles A, De L. Actitudes frente a la cultura de seguridad del paciente en el ámbito hospitalario y variables correlacionadas. Gac Sanit. 2017;31(2):145-149. doi:10.1016/j.gaceta.2016.07.019

39. Pineda, Ma. Acevedo, G. González Salinas, F. Castañeda Hidalgo H. Percepción de la cultura de enfermería, acerca de las fortalezas y oportunidades de mejora en la seguridad del usuario. 2013:1-11.

http://web.uaemex.mx/revistahorizontes/docs/revistas/Vol5/PERCEPCION_DE_LA_CULT URA_DE_ENFERMERIA.pdf.

40. Del O, Arco CDE. SOBRECARGA LABORAL EN PROFESIONALES DE ENFERMERÍA DE UNIDADES DE CUIDADO INTENSIVO EN INSTITUCIONES HOSPITALARIAS DE CARTAGENA DE INDIAS, 2012. tesis magister Univ Nac Colombi. 2013:89.

41. Pedro R, Antonio J, La EDE, et al. EL SÍNDROME DE QUEMARSE POR EL TRABAJO ( BURNOUT ). 2008.

42. Canales-vergara M, Paravic-klijn SVT. U n iv e r sit a ri a Condiciones de trabajo de los profesionales de enfermería en Chile. 2016;13(3):178-186.

43. Gherardi-donato CAB, Pereira ECS, Cardoso SS. Estrés laboral y estrategias de afrontamiento entre los profesionales de enfermería hospitalaria Estresse ocupacional e estratégias de enfrentamento entre profissionais de enfermagem em Occupational stress and coping strategies among nursing professionals. :288-298. 
44. Albendín L, Luis J, Ca GA, Ca GR. de Psicología Prevalencia bayesiana y niveles de burnout en enfermería de urgencias . Una revisión sistemática. 2016.

doi:10.1016/j.rlp.2015.05.004

45. Romero M, Lordui J, Pajaro C, Perez C. Relación entre la carga laboral de enfermería y la gravedad del paciente en unidades de cuidado intensivo de adultos.2011. revista aquichan disponible en: http://aquichan.unisabana.edu.co/index.php/aquichan/article/view/1899/2475 